\title{
Effects of Co-existence of Coronary Stenosis and the Extent of Coronary Ectasia on the TIMI Frame Count in Patients With Coronary Artery Ectasia
}

\author{
Feridun KosAr, ${ }^{1} \mathrm{MD}$, Nusret ACIKGOZ, ${ }^{1} \mathrm{MD}$, Ibrahim SAHIN,${ }^{2} \mathrm{MD}$, \\ Ergun TOPAL, ${ }^{1} \mathrm{MD}$, Hakan GUNEN, ${ }^{3} \mathrm{MD}$, Necip ERMIS, ${ }^{1} \mathrm{MD}$, \\ and Sengul CEHRELI, ${ }^{1} \mathrm{MD}$
}

\begin{abstract}
SUMMARY
The measurement of the thrombosis in myocardial infarction (TIMI) frame count is a simple method for evaluating coronary blood flow. Although it is well known that slow coronary flow is present in patients with coronary artery ectasia (CAE), the effects of coexisting stenosis and the severity of ectatic involvement on coronory flow have not been adequately studied. Thus, we examined (1) the effect of coexistence of obstructive coronary artery disease on TIMI frame count (TFC) and (2) the relation between the severity of ectatic involvement and TFC in patients with CAE. Ninety-seven study patients with CAE were examined in two steps to determine if they were appropriate in terms of the aim of this study. In the first step, ectasias were divided into three groups: an isolated CAE group, a CAE group with coexisting nonsignificant stenosis, and CAE with coexisting significant stenosis. In the second step, ectasias were subdivided into three groups: CAE with one segment, two segments, and three segments (or diffuse) involvement. The TIMI frame counts for the right coronary artery (RCA), the left circumflex coronary artery (LCx), and the left anterior descending artery (LAD) in the ectasia group were significantly higher than that of the control group $(P<0.001, P<0.05, P<0.05$, respectively). The presence of coexisting nonsignificant stenosis or coexisting significant stenosis in patients with CAE did not influence TFC $(P>0.05$, for all). In addition, the severity of ectasia involvement, regardless of the localization of ectatic segments and the type of the affected vessel, did not change the TFC $(P>0.05$, for all). These results suggest that neither the coexisting stenosis nor the extent of involvement significantly affect TFC in patients with coronary ectasia. (Int Heart J 2005; 46: 211-218)
\end{abstract}

Key words: Coronary artery ectasia, Coronary flow, TIMI frame count

CORONARY artery ectasia (CAE) is observed in 1 to $4 \%$ of patients undergoing coronary angiography, ${ }^{1-4)}$ and has been thought to be congenital or acquired. ${ }^{5-16)}$ The etiopathogenesis of this coronary enlargement is not very well known. How-

From the Departments of ${ }^{1}$ Cardiology, ${ }^{2}$ Internal Medicine, and ${ }^{3}$ Pulmonary Disease, Faculty of Medicine, Inonu University, Turgut Ozal Medical Center, Malatya, Turkey.

Address for correspondence: Feridun Kosar, MD, Department of Cardiology, Faculty of Medicine, Inonu University, 44069 Malatya, Turkey.

Received for publication January 13, 2004

Revised and accepted November 19, 2004. 
ever, extensive atherosclerotic changes and destruction of the media of the coronary artery vessels seem to play a role in the pathogenesis of CAE. ${ }^{8,9)}$

The thrombolysis in myocardial infarction (TIMI) frame count is a simple clinical tool for measuring and assessing coronary blood flow. Previous studies have showed that this measurement is significantly correlated with flow velocity measured with a flow-wire during baseline or hyperemia. Specifically, it has been shown that slow coronary flow status is present in coronary ectasia. ${ }^{17-20)}$ Several attempts have been made by using TIMI frame count to determine whether there is slow coronary flow in CAE. ${ }^{19,20)}$ To the best of our knowledge, there is only one study that has examined the effect of coexisting stenosis by means of the TIMI frame count method, ${ }^{21)}$ while no study has investigated the severity of ectasia involvement on slow coronary flow. Moreover, the study did not provide any detailed information about the relationship between TFC and the coexistence of obstructive coronary artery disease or the severity of ectatic involvement.

The present study was designed to elucidate how the coexistence of coronary stenosis and the severity of ectatic involvement influences coronary blood flow in patients with CAE using the TIMI frame count method.

\section{MeTHODS}

The study population included 97 patients with coronary artery ectasia (77 men, 20 women). The patients were selected from among 7200 patients undergoing coronary angiography between December 1998 and September 2003 in our department. The control group consisted of 35 patients with angiographically proven normal coronary arteries.

Evaluation of coronary artery ectasia by coronary angiography: Coronary angiography was routinely performed by the Judkins technique using 6-French right and left heart catheters (Integris H 3500 and 5000, Philips Medical Systems Turkey). At least five views of the left and two of the right coronary artery were recorded on 30 frames per second. Films of all patients with evidence of coronary ectasia were reviewed by three investigators, and a consensus was reached without knowledge of the clinical or laboratory findings.

Coronary artery ectasia was defined as dilation of the coronary artery to > 1.5 fold the diameter of the adjacent normal coronary vessels according to Falsetti and Carroll. ${ }^{6}$ In this study, we used the following anatomical classification of CAE in the evaluation of the extent of distribution of coronary artery ectasia. The coronary artery tree was subdivided into proximal, middle, and distal segments. For the left anterior descending artery (LAD), the vessel segments were defined as follows: the proximal segment was between the left main trunk and the first diagonal branch, the middle segment was between the first and second diag- 
onal branch, and the segment distal to the second diagonal branch was the distal segment of the LAD. In the same way, the proximal segment of the left circumflex coronary artery ( $\mathrm{LCx}$ ) was defined as the part of the vessel proximal to the first obtuse marginal branch, the median segment as the middle part between the first and second obtuse marginal branch, and the distal segment as the part distal to the second obtuse marginal branch. The corresponding segments in the right coronary artery (RCA) were defined according to the right ventricular branch and bifurcation of the posterior descending artery and the posterior left ventricular branch. According to the severity of ectatic involvement, ectasias were divided into three groups: CAE with one segment (group I), two segment (group II), and three segment (diffuse) involvement (group III). In addition, ectasias were subdivided depending upon the coexistence of coronary artery disease: isolated CAE (group I), CAE with coexisting stenotic lesions of $<50 \%$ (group II), and CAE with coexisting stenotic lesions of $>50 \%$ (group III).

Assessment of coronary blood flow: The TIMI frame count was determined for each major coronary artery in each patient according to methods first described by Gibson, et al. ${ }^{20)}$ The first frame is defined as the frame in which concentrated occupies the full width of the proximal coronary artery lumen, touching both borders of the lumen, and forward motion down the artery. The final frame is designated when the leading edge of the contrast column initially arrives at the distal landmark. The final frame used for the left anterior descending artery was the frame in which frame dye first entered the mustache segment. The final frame for the circumflex artery was found by using dye that first entered the distal bifurcation segment; this was the longest total distance from the coronary ostium. The frame in which dye first entered into the first branch of the posterolateral artery was used as the final frame for right coronary artery filling. Complete opacification of the target artery was not required for determining the final frame. Measurement of the frame count for each artery was then conducted by subtracting the first frame from the final frame. The LAD is usually longer than other major coronary arteries; the TFC for this vessel is often higher. The corrected left anterior descending artery frame rate was calculated by multiplying the frame rate by 1.7 as defined previously. The arteries that had values over the predicted normal frame counts were assessed as having slow coronary flow.

Statistical analysis: Data are presented as the mean \pm SD. The KolmogorovSmirnov test was performed to determine whether the data were normally distributed. This data was compared using an independent-samples T test. Categorical variables were compared using the chi-square test. The data in the three groups were compared by the Kruskal-Wallis variance analysis method as appropriate. A $P$ value $<0.05$ was considered statistically significant. 


\section{ReSults}

The patient characteristics for the ectasia and control groups are presented in Table I. No statistically significant differences were observed between the two groups with regards to age, gender, presence of hypertension and diabetes mellitus, or smoking $(P>0.05$ for all). The TIMI frame count of the RCA in the study group was significantly higher than that of the control group (53 \pm 14 versus $23 \pm$ $9, P<0.001)$. The TIMI frame counts of the study group for the LCx and the LAD were also significantly higher than those of the control group (for LCx $43 \pm$ 13 versus $23 \pm 7, P<0.05$; for LAD $45 \pm 16$ versus $25 \pm 8 ; P<0.05$ ) (Table II).

Coronary artery ectasia was found in $97(1.3 \%)$ of the patients undergoing coronary angiography. Fifty-nine patients had an isolated coronary ectasia (group I); the RCA in 31, LCx in 12, and LAD in 16. Twenty-one patients had coronary ectasia with nonsignificant stenosis (<50\%) (group II): the RCA in 9, LCx in 5, and LAD in 7. Sixteen patients had coronary ectasia with significant stenosis (> $50 \%$ ) (group III): the RCA in 7, LCx in 4, and LAD in 6. TIMI frame counts were compared among the groups to demonstrate whether coexisting nonsignificant and significant stenosis influences TFC (Table III). There were no statistically significant differences in TIMI frame counts among these subgroups.

Table I. Clinical Characteristics of Patients with Coronary Artery Ectasia and Subjects With Normal Coronary Arteries

\begin{tabular}{lccc}
\hline & Ectasia group $(n=97)$ & Control group $(n=35)$ & $P$ value \\
\hline Age (years) & $58 \pm 10$ & $55 \pm 7$ & NS \\
Men/women & $77 / 20$ & $25 / 10$ & NS \\
SBP (mmHg) & $132 \pm 23$ & $127 \pm 14$ & NS \\
DBP (mmHg) & $82 \pm 12$ & $79 \pm 10$ & NS \\
Diabetes mellitus & $11 / 97$ & $3 / 35$ & NS \\
Smoking & $35 / 97$ & $11 / 35$ & NS \\
\hline
\end{tabular}

Values are mean \pm SD. NS = not significant; SBP = systolic blood pressure; $\mathrm{DBP}=$ diastolic blood pressure.

Table II. TIMI Frame Counts for Each Coronary Artery of Patients With Coronary Ectasia and Control Subjects

\begin{tabular}{lccc}
\hline & $\begin{array}{c}\text { Ectasia group } \\
n=97\end{array}$ & $\begin{array}{c}\text { Control group } \\
n=35\end{array}$ & $P$ value \\
\hline Right coronary artery & $53 \pm 14(n=47)$ & $23 \pm 9(n=11)$ & $<0.001$ \\
Circumflex artery & $43 \pm 13(n=21)$ & $23 \pm 7(n=12)$ & $<0.05$ \\
$*$ Left anterior descending artery & $45 \pm 16(n=29)$ & $25 \pm 8(n=12)$ & $<0.05$ \\
\hline Values are mean \pm SD. & & \\
$*$ The corrected TIMI frame count is shown for the left anterior descending artery.
\end{tabular}


Table III. TIMI Frame Counts in Patients With Isolated CAE (Group I), CAE With Coexisting Nonsignificant Stenosis (Group II), and CAE With Coexisting Significant Stenosis (Group III) in the Right, Circumflex, and Left Anterior Descending Coronary Arteries

\begin{tabular}{lcccc}
\hline & Group I $(n=59)$ & Group II $(n=21)$ & Group III $(n=17)$ & $P$ \\
\hline Right coronary artery & $51 \pm 14(n=31)$ & $55 \pm 15(n=9)$ & $59 \pm 19(n=7)$ & $>0.05 \alpha, \beta$ \\
Circumflex artery & $40 \pm 13(n=12)$ & $44 \pm 15(n=5)$ & $50 \pm 17(n=4)$ & $>0.05 \alpha, \beta$ \\
*Left anterior descending artery & $42 \pm 11(n=16)$ & $47 \pm 12(n=7)$ & $51 \pm 15(n=6)$ & $>0.05 \alpha, \beta$ \\
\hline
\end{tabular}

Values are mean \pm SD.

$\alpha$ : Difference between group I and group III

$\beta$ : Difference between group II and group III

*The corrected TIMI frame count is shown for the left anterior descending artery.

Table IV. TIMI Frame Counts in Patients With CAE With One Segment (Group I), Two Segment (Group II) and Three Segment Involvement (Group III) in the Right, Circumflex, and Left Anterior Descending Coronary Arteries

\begin{tabular}{lcccc}
\hline & Group I $(n=9)$ & Group II $(n=19)$ & Group III $(n=31)$ & $P$ value \\
\hline Right coronary artery & $48 \pm 7(n=4)$ & $51 \pm 12(n=10)$ & $55 \pm 17(n=16)$ & $>0.05 \alpha, \beta$ \\
Circumflex artery & $33 \pm 10(n=2)$ & $35 \pm 13(n=4)$ & $39 \pm 14(n=7)$ & $>0.05 \alpha, \beta$ \\
*Left anterior descending artery & $38 \pm 9(n=3)$ & $41 \pm 11(n=5)$ & $44 \pm 15(n=8)$ & $>0.05 \alpha, \beta$
\end{tabular}

Values are mean $\pm \mathrm{SD}$.

$\alpha$ : Difference between group I and group III

$\beta$ : Difference between group II and group III

*The corrected TIMI frame count is shown for the left anterior descending artery.

In the present study, 9 of 59 patients with isolated coronary ectasia (15.2\%) had only one ectatic segment, 19 had two ectatic segment, and 31 had three segment involvement. TIMI frame counts were compared among these groups to demonstrate the alteration of coronary flow according to the severity of ectatic involvement in CAE. TIMI frame counts were larger in those with two segment involvement (group II) than in coronary ectasia with one segment involvement (group I), and larger in those with three segment involvement (diffuse) (group III) than in coronary ectasia with two segment involvement. However, none of these differences between these groups was statistically significant $(P>0.05)$ (Table IV).

\section{DISCUSSION}

Hartnell, et $a l^{1)}$ used the term ectasia to define abnormal dilation of coronary segments 1.5 -fold or more of the adjacent normal segment. Coronary artery dilation may be localized or diffuse with or without coronary artery stenosis. The incidence of coronary artery ectasia in patients undergoing angiography has been 
reported as $0.3-4.9 \%$, regardless of coexisting coronary artery stenosis. ${ }^{1-4)}$ Most studies have shown that underlying cytological changes are identical to those found in atherosclerotic lesions. The majority of CAE are due to coronary artery atherosclerosis. $^{5-9)}$ In $30 \%$ to $40 \%$, CAE is associated with congenital coronary abnormalities $^{10,11)}$ and connective tissue diseases. ${ }^{12-16)}$

Kruger, et $a l^{17)}$ have postulated that altered coronary flow in CAE may induce myocardial ischemia and infarction. Hamaoka, et al ${ }^{19)}$ found by means of a Doppler guide wire significantly reduced coronary flow velocity in patients with Kawasaki disease. These measurements have been significantly correlated with coronary flow velocity measured with a Doppler guide wire by several investigators. ${ }^{18,19)}$ Gibson, et $a l^{20)}$ described the Thrombolysis In Myocardial Infarction (TIMI) frame count method for measuring coronary flow velocity from coronary angiograms. In the literature, the phenomenon of coronary slow flow is well known for coronary artery ectasia. ${ }^{17-19)}$ It has been speculated that slow flow is aggravated with the increasing severity of ectasia involvement. However, the relationship between coexisting nonobstructive stenosis and the severity of ectatic involvement and slow flow has not been well documented by means of the TIMI frame count.

The overall incidence of CAE in this study was similar to that reported in previous studies. CAE was most frequently found in the right coronary artery and CAE tended to involve one and two vessels. Also, the TIMI frame counts for each coronary artery in the ectasia group were significantly higher than those of patients with angiographically normal coronary arteries. Recently, Papadakis, et $a l^{21)}$ documented slow coronary flow in patients with CAE by using the TIMI frame count method. Also they further demonstrated that the right coronary artery ectasias with coexisting obstructive disease had the slowest coronary flow. In this study, we evaluated the effects of coexisting nonsignificant stenosis and significant stenosis together on TFC in patients with CAE. When ectasia coexisted with nonsignificant stenosis or significant stenosis, the TIMI frame counts were not different from that of coronary ectasia. In other words, the presence of the coexisting nonsignificant stenosis or significant stenosis did not further impair flow velocity in patients with coronary ectasia. Additionally, we demonstrated that the severity of ectasia involvement, regardless of the localization of ectatic segments and the type of affected vessel, did not change the TFC. These findings suggest that the coexisting stenosis and the severity of ectasia involvement may not be strongly related to TIMI frame count. Although we cannot explain the underlying mechanisms precisely, these data indicate that, in addition to the coexisting stenosis and the severity of ectasia involvement, increased microcirculation resistance observed in coronary ectasia and coronary artery disease can affect TIMI frame count in patients with CAE. 
Study limitations: The TFC provides a more objective assessment of coronary flow, but it is a complex measurement that is related to clinical and angiographic variables. Also, it has been suggested that TFC is an index of microvascular behavior, which reflects the coronary vascular resistance. Many metabolic, neurohumoral, and hemodynamic factors can affect coronary vascular resistance, and thus alter the TFC. Therefore, it is not possible to say how the coexisting stenosis and the severity of ectasia involvement influence the TFC in patients with CAE. Conclusions: This is the first study using the TIMI frame count method to indicate that coexisting coronary stenosis and the severity of ectasia involvement did not further impair the TFC, contrary to expectations. This study has provided the first quantitative data addressing the relationship between these parameters and TFC. Further studies are required to validate our findings.

\section{REFERENCES}

1. Hartnell GG, Parnell BM, Pridie RB. Coronary artery ectasia. Its prevalence and clinical significance in 4,993 patients. Br Heart J 1985; 54: 392-5.

2. Markis JE, Joffe CD, Cohn PF, Feen DJ, Herman MV, Gorlin R. Clinical significance of coronary arterial ectasia. Am J Cardiol 1976; 37: 217-22. (Review)

3. Oliveros RA, Falsetti HL, Carroll RJ, Heinle RA, Ryan GF. Atherosclerotic coronary artery aneurysm. Report of five cases and review of the literature. Arch Intern Med 1974; 134: 1072-6.

4. Swaye PS, Fisher LD, Litwin P, et al. Aneurysmal coronary artery disease. Circulation 1983; 67: 134-8.

5. Seabra-Gomes R, Somerville J, Ross DN, Emanuel R, Parker DJ, Wong M. Congenital coronary artery aneurysms. Br Heart J 1974; 36: 329-35.

6. Falsetti HL, Carroll RJ. Coronary artery aneurysm. A review of the literature with a report of 11 new cases. Chest 1976; 69: 630-6.

7. Swanton RH, Thomas ML, Coltart DJ, Jenkins BS, Webb-Peploe MM, Williams BT. Coronary artery ectasia a variant of occlusive coronary arteriosclerosis. Br Heart J 1978; 40: 393-400.

8. Michael JA Williams, Ralph AH Stewart. Coronary artery ectasia: Local pathology or diffuse disease? Cathet Cardiovasc Diagn 1994; 33: 116-9.

9. Befeler B, Aranda MJ, Embi A, Mullin FL, El-Sherif N, Lazzara R. Coronary artery aneurysms: study of the etiology, clinical course and effect on left ventricular function and prognosis. Am J Med 1977; 62: 597-607.

10. Yamanaka O, Hobbs RE. Coronary artery anomalies in 126,595 patients undergoing coronary arteriography. Cathet Cardiovasc Diagn 1990; 21: 28-40.

11. Vieweg WVR, Alpert JS, Hagen AD. Caliber and distribution of normal coronary arterial anatomy. Cathet Cardiovasc Diagn 1976; 2: 269-80.

12. Demopoulos VP, Olympios CD, Fakiolas CN, et al. The natural history of aneurysmal coronary artery disease. Heart 1977; 78: 136-41.

13. Breeker SJ, Gray HH, Oldershaw PJ. Coronary artery aneurysms and myocardial infarction: adult sequelae of Kawasaki disease? Br Heart J 1988; 59: 509-12.

14. Sheikhzadeh A, Ghabussi P. A survey of uncommon forms of coronary arteries. Jpn Heart J 1982; 23: 725-31.

15. Kurokawa H, Kondo T, Shiga, et al. Myocardial infarction due to thrombi in coronary aneurysms in a young woman with systemic lupus erythematosus. J Cardiogr 1986; 16: 249-58. (Japanese)

16. DiMaria C, Zanchetta M, Maiolino P. Coronary aneurysms in a case of Ehlers-Danlos syndrome. Jpn Heart J 1988; 29: 491-6.

17. Kruger D, Stierle U, Herrmann G, Simon R, Sheikhzadeh A. Exercise-induced myocardial ischemia in isolated coronary artery ectasias and aneurysms (“dilated coronopathy”). J Am Coll Cardiol 1999; 34: 1461-70. 
18. Akyurek O, Berkalp B, Sayin T, Kumbasar D, Kervancioglu C, Oral D. Altered coronary flow properties in diffuse coronary artery ectasia. Am Heart J 2003; 145: 66-72.

19. Hamaoka K, Onouchi Z, Kamiya Y, Sakata K. Evaluation of coronary flow velocity dynamics and flow reserve in patients with Kawasaki disease by means of a Doppler guide wire. J Am Coll Cardiol 1998; 31: 833-40.

20. Gibson CM, Cannon CP, Daley WL, et al. TIMI frame count: a quantitative method of assessing coronary artery flow. Circulation 1996; 93: 879-88.

21. Papadakis MC, Manginas A, Cotileas P, et al. Documentation of slow coronary flow by the TIMI frame count in patients with coronary ectasia. Am J Cardiol 2001; 88: 1030-2. 